Transcontinentales

continentales

Sociétés, idéologies, système mondial

La transition chinoise

\title{
L'éducation en Chine à l'ère des réformes
}

Michel Grenié et Agnès Belotel-Grenié

\section{(2) OpenEdition}

\section{Journals}

Édition électronique

URL : http://journals.openedition.org/transcontinentales/544

DOI : 10.4000/transcontinentales.544

ISBN : 978-2-8218-1112-6

ISSN : 1775-397X

\section{Éditeur}

Editions de la maison des sciences de l'homme

Édition imprimée

Date de publication : 31 décembre 2006

Pagination : $67-85$

ISBN : 978-2-200-92170-5

ISSN : $1950-1684$

Référence électronique

Michel Grenié et Agnès Belotel-Grenié, «L'éducation en Chine à l'ère des réformes », Transcontinentales [En ligne], 3 | 2006, document 4, mis en ligne le 12 octobre 2011, consulté le 08 septembre 2020. URL: http://journals.openedition.org/transcontinentales/544; DOI : https://doi.org/10.4000/ transcontinentales.544

Ce document a été généré automatiquement le 8 septembre 2020

Tous droits réservés 


\title{
L'éducation en Chine à l'ère des réformes
}

\author{
Michel Grenié et Agnès Belotel-Grenié
}

1 Juin 1989, un étudiant chinois se dresse face à un char place Tiananmen : le monde entier découvre une jeunesse pleine d'espoir mobilisée par le devoir de faire évoluer la société et avancer les idéaux de liberté et de démocratie. Juin 2006, plusieurs milliers d'étudiants de l'université de Zhengzhou (province centrale du Henan) manifestent violemment sur leur campus dans la nuit du 15 : ils ont appris que le diplôme qui leur sera délivré mentionne seulement le nom de leur institut privé et non celui - plus prestigieux - de l'université publique qui les héberge ${ }^{1}$. Entre ces deux mouvements, une génération s'est écoulée : l'idéalisme de 1989 a laissé la place à des préoccupations plus individualistes centrées sur l'avenir de chacun dans une société de plus en plus marquée par la compétition et la marchandisation. Les questions matérielles ont pris le pas sur les questions politiques. Beaucoup de familles s'endettent pour financer, dès le primaire ou le collège, leçons particulières ou cours d'été, et sont prêtes à payer très cher une inscription dans un établissement réputé afin d'accroitre les chances de leur enfant de réussir le concours national avec un score qui ouvre aux universités d'élite dont les diplômes assurent les meilleures positions sociales.

2 En moins de trente ans de politique d'ouverture et de réforme économique, le paysage social de la Chine a énormément changé. Le passage d'une économie planifiée à "l'économie socialiste de marché »- concept forgé par les autorités pour évacuer le politique et indiquer que prospérité et renforcement de la Chine sont l'objectif exclusif des réformes dans un cadre où le parti unique continue à exercer le monopole du pouvoir - s'est traduit par l'évolution rapide des formes d'emploi et -d'organisation sociale : la culture bureaucratique où tout le monde bénéficiait peu ou prou, à travers une gestion collective, du même salaire et de garanties sociales à vie, a laissé la place à un univers où la mise en concurrence devient systématique et rime avec une gestion individualisée du monde du travail. La restructuration des entreprises d'État, initiée au milieu des années 1990, a liquidé la classe ouvrière en introduisant une gestion fluide et flexible de la main-d'œuvre et l'abandon du fardeau de la protection sociale : c'est la 
" mise au travail capitaliste de la Chine ${ }^{2}$ ». La mondialisation et l'adhésion de la Chine à l'Organisation mondiale du commerce n'ont fait qu'accélérer le processus de marginalisation du travail productif et de précarisation des emplois les moins qualifiés.

3 La Chine continue à investir fortement dans les industries à faible valeur ajoutée très consommatrices de main-d'œuvre. Face à un risque de surchauffe économique lié à un surinvestissement dans les capacités de production, il est essentiel de développer des emplois plus qualifiés et plus flexibles qui accordent davantage de place au travail immatériel et aux technologies de la connaissance. Moderniser le système éducatif est indispensable pour former des salariés hautement qualifiés, accroître la part des activités tertiaires dans la richesse nationale, renforcer la valorisation du capital humain ainsi que la recherche et l'innovation ${ }^{3}$.

Cet article dresse un tableau des grands changements qui ont marqué le système éducatif chinois de 1978 à 2006. Si les progrès accomplis sont importants sur de nombreux points (réduction de l'illettrisme, généralisation de l'enseignement obligatoire, essor de l'enseignement privé, massification de l'enseignement supérieur), il n'en demeure pas moins que le financement public de l'éducation reste insuffisant pour compenser les écarts de revenus entre les régions riches et les régions pauvres. Le coût des études est très lourd pour les familles rurales. Le bilan des réformes est donc contrasté : massification mais accès inégalitaire aux études selon le statut social des familles et leur lieu de résidence, exigence de réussite individuelle qui bute sur les mots d'ordre socialistes rabâchant que le collectif prime sur l'individuel, ressources éducatives abondantes des régions côtières développées ou urbanisées face aux régions rurales de l'Ouest déshéritées, émergence d'un secteur privé dynamique alors que le profit dans ce domaine est considéré avec suspicion, ouverture à la pédagogie occidentale davantage centrée sur l'apprenant tout en revendiquant les spécificités de l'héritage confucéen, besoin de renforcer la créativité et l'innovation alors que le Parti communiste s'en tient à une orthodoxie de marbre qui récuse toute critique.

\section{Une vision politique et sociale de l'éducation}

5 La Chine a depuis fort longtemps accordé un rôle fondamental à l'éducation. Comme le souligne Jacques Gernet ${ }^{4}$, l'éducation est depuis les Han un des fondements de l'ordre politique et de l'harmonie sociale. Confucius (551 à 479 av. J.-C.) est sans doute celui qui a le plus marqué la pensée chinoise sur l'éducation. Ses enseignements insistent sur le besoin d'harmonie dans la société : vivre en harmonie avec les autres, aussi bien dans la famille - qui constitue le modèle naturel par excellence - que dans le reste de la société. C'est par une éducation fondée sur l'exemple que se transmettent les cinq vertus morales, piliers de l'établissement et du maintien d'une harmonie sociale respectueuse des relations hiérarchiques : ren (la bienveillance), yi (la droiture), li (la bienséance), zhi (la sagesse), xin (la loyauté). Cette éducation a comme idéal l'intégration sociale ${ }^{5}$. Sa finalité première est de doter chaque esprit d'une conscience sociale développée et d'inculquer les codes qui, à travers le respect des aînés et la piété filiale, garantissent la stabilité sociale. Les maitres de l'époque classique associèrent intimement réflexion sur l'art de gouverner et enseignement : Confucius, Mo Zi, Mencius, Xun Zi, Lao Zi, Zhuang $\mathrm{Zi}$, Shang Yang et Han Fei fondèrent des écoles et eurent de nombreux disciples. Cela explique qu'enseignants et lettrés jouissent d'une place privilégiée vis-à-vis des gouvernants. Le système éducatif a une double mission politique : élever les qualités 
morales de la population et identifier les individus loyaux et talentueux aptes au service de l'État ${ }^{6}$. Le système des concours mandarinaux fut en vigueur pendant près de mille trois cents ans et ne prit fin qu'en 1905: son contenu et son organisation contribuèrent largement a renforcer le socle des valeurs confucéennes gages de l'unité de la société. Il explique en grande partie la longévité du système impérial : « Tout l'art de la réussite consistait à apprendre par cœur et à savoir imiter les modèles de composition ${ }^{7}$.» Le succès aux examens était pour les intéressés et leurs familles l'assurance d'une carrière garantissant des avantages matériels et une grande reconnaissance sociale ${ }^{8}$. À l'époque impériale, tout bon gouvernant -s'efforce de s'appuyer sur l'éducation pour asseoir son autorité et éviter les désordres. L'éducation et la culture furent instrumentalisées par les empereurs et leurs conseillers pour renforcer l'absolutisme du pouvoir et rendre toute alternative au despotisme impensable : Jean-François Billeter souligne que ce que l'on appelle de nos jours «la civilisation chinoise " résulte de la mise en place systématique et progressive du « canon confucianiste » dont la première finalité fut d'occulter la nature totalitaire du pouvoir 9 .

6 En accordant, dès le début de sa stratégie de conquête du pouvoir, un rôle prééminent à l'éducation politique, Mao Zedong s'inscrit dans la continuité. Pour lui l'émergence d'un ordre nouveau lié à l'implantation du Parti communiste chinois (PCC) dans les campagnes passe par l'éducation des paysans ${ }^{10}$. L'éducation est au service de l'édification du socialisme et de la lutte des classes. En 1957, pour renforcer la place du marxisme dans l'éducation, le Comité central affecte un millier de cadres du Parti dans les établissements. En 1958, la « Directive concernant -l'action éducative »-promulguée par le Comité central du Parti communiste et le Conseil des affaires -d'État souligne que l'éducation est au service de la politique prolétarienne et qu'elle doit être combinée avec le travail productif ${ }^{11}$. La Révolution culturelle (1966-1976) marque l'apogée de cette approche défendue par l'aile gauche du Parti : universités et laboratoires de recherche sont fermés; étudiants, enseignants et chercheurs sont par millions envoyés en la campagne pour - selon la terminologie maoïste de l'époque - «parfaire », au contact des paysans et du travail productif, leur conscience politique. Lorsqu'en 1978, de retour au pouvoir, Deng Xiaoping lance sa politique d'ouverture et de réforme reposant sur les quatre modernisations (agriculture, industrie, sciences et technologies, défense nationale), il rompt avec l'aile gauche du Parti et rappelle de façon pragmatique le lien nécessaire entre le progrès économique et social et le développement de l'éducation, des sciences et des technologies.

7 Ce rappel historique éclaire pourquoi, encore aujourd'hui, l'enseignement en Chine est souvent moins tourné vers l'acquisition de connaissances ou de savoirs pratiques que vers celle de valeurs morales et sociales. Les examens continuent à y jouer un rôle central: «La Chine qui a inventé la religion du diplôme et les examens n'est jamais parvenue à s'en libérer ${ }^{12}$. » Néanmoins, au fur et à mesure que se développaient « l'économie socialiste de marché » et le secteur privé, le système éducatif chinois a dû évoluer, notamment l'enseignement supérieur: le contenu des formations s'est orienté davantage vers des contenus pratiques et a tenté de répondre aux besoins des entreprises. Pour autant, le contrôle politique du PCC ne s'est pas relâché : l'étude du marxisme-léninisme et celle de la pensée de Mao Zedong demeurent obligatoires pour tous. Toutefois, au fur et à mesure que la massification et la marchandisation de l'enseignement secondaire et supérieur se poursuivent, certaines contradictions liées à l'héritage du système traditionnel - 
deviennent de plus en plus flagrantes : assurer l'harmonie de la société et l'unité nationale ne rime pas nécessairement avec développement personnel et innovation scientifique ou technologique. L'introduction récente du concept de « société socialiste harmonieuse » et le rappel de la nécessité de « promouvoir la morale au sein du peuple » montre la volonté des dirigeants chinois de penser la modernisation de leur pays à travers un retour à ses racines confucéennes ${ }^{13}$.

\section{Présentation générale du système éducatif chinois}

En 2004, le système éducatif chinois formait 261,16 millions d'individus, $(20 \%$ de la population) grâce à un personnel éducatif de 15,97 millions de personnes dont 12,76 millions d'enseignants ${ }^{14}$. Il se découpe comme suit ${ }^{15}$ :

- - enseignement préscolaire non obligatoire pour les enfants de plus 3 ans (durée trois ans);

- - enseignement obligatoire pour les enfants de 7 à 15 ans (neuf ans selon un découpage six ans de primaire et trois ans de $1^{\text {er }}$ cycle du secondaire ou, plus rarement, cinq et quatre ans) ;

- - enseignement secondaire du 2 cycle (lycée) pour les jeunes 15 à 18 ans, qui se subdivise en formation générale (trois ans) accessible sur concours ou formations spécialisées et professionnelles ou techniques (trois à cinq ans) ;

- - enseignement supérieur au-delà de 18 ans, assuré soit dans des établissements supérieurs d'enseignement général (instituts ou universités) délivrant des formations courtes dazhuan (trois ans) et des licences générales benke (quatre ans) ouvrant à des masters (trois ans) puis à des doctorats (trois ans), soit dans des instituts supérieurs techniques et professionnels délivrant des formations courtes (deux à trois ans).

9 La formation secondaire générale du 2 cycle a la préférence des familles car elle ouvre à la poursuite d'études supérieures pour ceux qui réussissent le concours national d'accès à l'université $(\text { gaokao })^{16}$. Elle est d'ailleurs axée presque exclusivement sur la préparation de ce concours. À l'université, le passage de licence en master, tout comme celui de master en doctorat, est conditionné par la réussite d'un concours très sélectif. Un important secteur de formation pour adultes est présent au niveau secondaire et supérieur.

10 À partir de 1995, date à laquelle a été publiée la loi sur l'éducation fixant les grandes lignes de la réforme de l'éducation, le taux de scolarisation en primaire et en collège s'est accru régulièrement pour atteindre, en 2004, 98,9\% pour le primaire et $94,1 \%$ pour le collège (voir figure 1 et tableau 1$)^{17}$. La baisse des effectifs du primaire, à partir de 1999, et celle du collège, six ans plus tard, s'expliquent pour des raisons démographiques et devraient se poursuivre. Le taux de scolarisation en maternelle progresse régulièrement mais reste encore faible ( $40,75 \%$ en 2004).

Les progrès en faveur de l'enseignement obligatoire de neuf ans sont nets. En 2004, $85 \%$ de la population chinoise avait suivi une scolarisation obligatoire de neuf ans et la durée moyenne de scolarisation de la population active ( 15 ans et plus) était de 8,3 années ; le taux d'illettrés et de quasi-illettrés dans la population âgée de 15 ans et plus était de $10,32 \%$ (soit 104 millions de personnes), contre 22,23\% lors du recensement de 1990. Enfin, en 2004, le taux d'illettrisme dans la population des 15-50 ans est passé sous la barre des $4 \%$. 
Fig 1

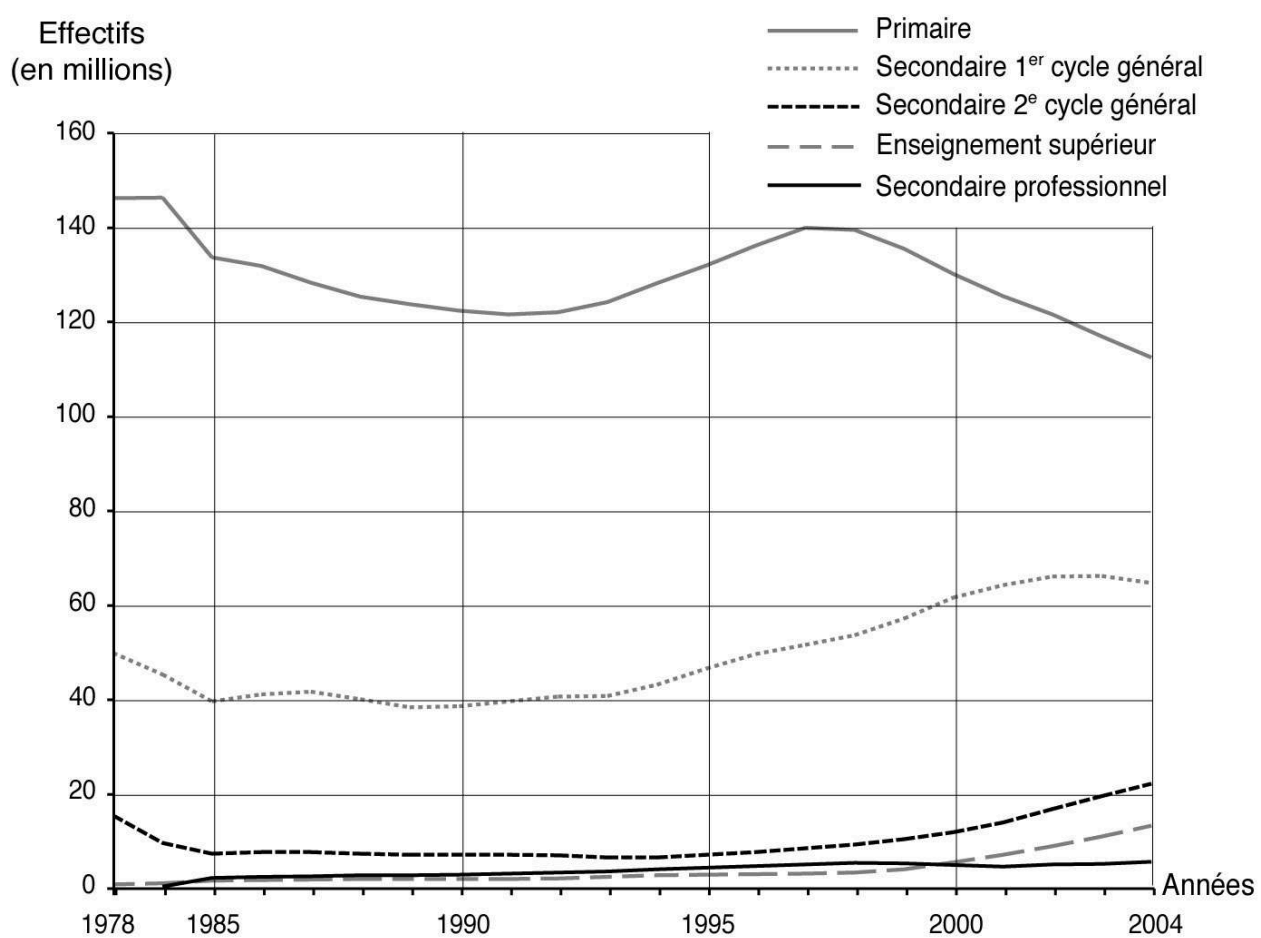

Effectifs scolaires et universitaires selon les cycles d'étude

Tableau 1

\begin{tabular}{|c|c|c|c|c|c|}
\hline & $\begin{array}{c}\text { Primaire } \\
\text { (de 6 à 11 ans) }\end{array}$ & $\begin{array}{c}\text { Secondaire } \\
\text { ler cycle } \\
\text { général } \\
\text { (de 12 à 14 ans) }\end{array}$ & $\begin{array}{r}\text { Secondaire } \\
\text { 2e cycle } \\
\text { général } \\
\text { (de 15 à } 17 \\
\text { ans) }\end{array}$ & $\begin{array}{c}\text { Enseignement } \\
\text { supérieur } \\
\text { (18 ans et plus) }\end{array}$ \\
\hline $\mathbf{1 9 9 0}$ & 96,3 & 66,7 & 21,9 & 26,0 & 3,4 \\
\hline $\mathbf{1 9 9 5}$ & 98,5 & 78,4 & 28,8 & 33,6 & 7,2 \\
\hline $\mathbf{1 9 9 8}$ & 98,9 & 87,3 & 34,4 & 40,7 & 9,8 \\
\hline $\mathbf{1 9 9 9}$ & 99,1 & 88,6 & 35,8 & 41,5 & 10,5 \\
\hline $\mathbf{2 0 0 0}$ & 99,1 & 88,6 & 38,2 & 42,8 & 11,5 \\
\hline $\mathbf{2 0 0 1}$ & 99,1 & 88,7 & 38,6 & 42,8 & 13,3 \\
\hline $\mathbf{2 0 0 2}$ & 98,6 & 90,0 & 38,4 & 42,8 & 15,0 \\
\hline $\mathbf{2 0 0 3}$ & 98,7 & 92,7 & 42,1 & 43,8 & 17,0 \\
\hline
\end{tabular}




\begin{tabular}{|l|l|l|l|l|l|}
\hline 2004 & 98,9 & 94,1 & 46,5 & 48,1 & 19,0 \\
\hline
\end{tabular}

Évolution du taux de scolarisation selon le cycle d'étude

Depuis 1999, les autorités ont accentué leurs efforts en faveur des lycées et du supérieur. En 2004, 62,9\% des élèves sortant du collège ont continué leurs études en lycée en réussissant le concours d'admission; les formations générales accueillaient $61,11 \%$ des lycéens contre $38,3 \%$ pour les filières techniques, spécialisées et professionnelles. Cette même année, près de $82,5 \%$ des diplômés du secondaire ont été admis dans le supérieur. Les efforts considérables consentis pour l'enseignement supérieur ont permis de passer de 3,4 millions d'étudiants en 1998 à 14,15 millions en $2004^{18}$. En 2006, près de 8,8 millions de candidats ont présenté le gaokao avec un taux d'admission proche de $50 \%$.

\section{Les grandes réformes}

\section{Aspects législatifs de l'éducation}

13 L'article 19 de la Constitution de $1982^{19}$ fixe que «l'État développe l'éducation socialiste pour élever le niveau culturel et scientifique de tout le peuple». Plusieurs lois visant "à revivifier le pays au moyen de la science et de l'éducation" le complètent ${ }^{20}$ et rappellent que l'éducation est au service « de la modernisation socialiste » et a pour objectif de «bâtir une société socialiste avancée culturellement et idéologiquement ainsi que matériellement». L'éducation repose sur "les principes du marxisme, du léninisme, de la pensée de Mao Zedong et de la théorie de la construction d'un socialisme aux caractéristiques chinoises ». C'est pourquoi, la formation idéologique et politique des jeunes est omniprésente à chaque étape du parcours éducatif.

14 Les principales dispositions sont résumées dans les lignes qui suivent. Tout enfant âgé d'au moins 6 ans doit suivre une scolarité obligatoire de neuf ans. Les gouvernements locaux sont responsables, sous le contrôle des autorités centrales, de cette période de formation (en théorie) gratuite. L'État veille à inculquer aux élèves et aux étudiants «le patriotisme, le collectivisme, le socialisme ainsi que les valeurs liées au respect de la loi, à la défense de la patrie et à l'unité nationale ». La religion n'a pas sa place dans les établissements. Le chinois standard est la langue adoptée sauf quand les élèves des minorités nationales sont majoritaires. Sous le contrôle du Conseil d'État ${ }^{21}$, les gouvernements locaux gèrent l'enseignement primaire et secondaire. L'enseignement supérieur relève du gouvernement central et des gouvernements provinciaux. L'État s'est engagé à développer l'enseignement professionnel dans les zones rurales et à l'ouvrir aux femmes, aux handicapés ainsi qu'aux chômeurs. Les gouvernements locaux des districts et des niveaux supérieurs, sous l'égide de la section du Conseil d'État en charge de l'enseignement professionnel, coordonnent cet enseignement. L'enseignement non gouvernemental et privé est une composante du «système d'éducation socialiste ». 


\section{Un financement public insuffisant}

15 L'essor économique dans les années 1980 a conduit à une réforme des finances publiques qui répartit les rôles entre les différents niveaux de gouvernement (central, provincial, district, ville, village). La loi de 1986 sur l'enseignement obligatoire a décentralisé les responsabilités dans ce cadre ${ }^{22}$. Elle visait à élargir les sources de financement et à responsabiliser les dirigeants locaux en introduisant des mécanismes d'économie de marché qui les pousseraient à prendre des initiatives pour financer les établissements de leur secteur. Le gouvernement central fixe les choix stratégiques pour l'organisation et le pilotage du système éducatif; il focalise son soutien sur l'enseignement supérieur et certains projets spécifiques. Le gouvernement provincial assure le contrôle des directives du ministère de l'Éducation et veille à leur mise en œuvre. Il finance les établissements supérieurs qui relèvent du niveau provincial. Le district et les niveaux inférieurs sont responsables de l'organisation matérielle et humaine de l'enseignement primaire et secondaire. Ils mobilisent les autorités locales et les autres partenaires locaux pour assurer les budgets requis pour l'éducation de base ainsi que pour les lycées et la rémunération de leur personnel : taxes locales en faveur de l'éducation, fonds des entreprises des établissements scolaires ou d'autres entreprises, frais de scolarité à partir du lycée et autres frais à la charge des familles dès le primaire pour les livres, les examens ou les cours optionnel, donations, etc. Les villes financent les collèges et leur personnel. Les écoles primaires sont prises en charge, selon leur implantation, par les villes ou les villages. Les villages qui ne disposent pas de ressources suffisantes peuvent ponctuellement être soutenus par le gouvernement central. Les bâtiments, leur sécurité, leur équipement ainsi que le recrutement, la promotion et la rémunération du personnel relèvent désormais des échelons locaux.

16 Pour autant, face à l'étendue des besoins, l'effort public de financement de l'éducation reste largement insuffisant : en 2004, le budget public alloué à l'éducation, tous niveaux confondus, atteignait 724,26 milliards de yuans $(+15,98 \%$ par rapport à 2003$)$, soit $2,79 \%$ du produit intérieur brut (PIB ${ }^{23}$, un chiffre bien inférieur à la moyenne mondiale des pays en voie de développement $(3,9 \%)$ et à celle des pays développés $(5 \%)$. Cette somme ne couvre plus que $62 \%$ du financement total de l'éducation: les familles contribuent à financer $18 \%$ du coût de l'éducation ${ }^{24}$. Entre 1992 et 2004 le budget public a quintuplé tandis que la part demandée aux familles était multipliée par vingt-cinq. L'éducation est devenue pour les familles le second poste de dépense après le logement. Les familles rurales consacrent 32,6\% de leur revenu annuel à l'éducation contre 25,9\% pour les familles urbaines ${ }^{25}$.

17 Face à un financement public de l'enseignement primaire et secondaire notoirement insuffisant, la priorité accordée à l'enseignement supérieur paraît discutable. Désireuses, par nationalisme, de renforcer la place des universités chinoises dans la course mondiale à l'excellence, les autorités ont privilégié ces dernières années le budget de l'enseignement supérieur. En 2003, il accaparait 22,8\% du budget total de l'éducation $(28,89 \%$ en 2002$)$ pour environ 14 millions d'étudiants et 7 millions d'adultes, alors que $31 \%(38,41 \%$ en 2002$)$ étaient consacrés au secondaire pour 102 millions d'élèves et $33 \%(26,43 \%$ en 2002$)$ pour le primaire qui comptait 116 millions d'élèves. 


\section{Des disparités croissantes}

18 La décentralisation du financement de l'enseignement obligatoire grève largement le budget des villages pauvres qui consacrent l'essentiel de leur soutien aux salaires des enseignants, au détriment des investissements immobiliers ou du matériel pédagogique. Puisque la loi de 1986 interdisait la perception de frais d'inscription durant les neuf années d'enseignement obligatoire, les établissements ont exigé des familles, pour boucler leur budget, le paiement de frais divers (d'examen, d'uniforme, de chauffage, de literie...) qui correspondaient parfois dans les campagnes à plusieurs mois de salaires, et ils ont développé en leur sein des entreprises bénéficiant d'exonérations fiscales ${ }^{26}$. Le monde entier a découvert cette triste réalité à travers le drame survenu le 6 mars 2001 dans une école du village de Fanglin, dans la province du Jiangxi, où trente-sept enfants et cinq adultes furent tués par l'explosion de feux d'artifice qu'ils assemblaient ordinairement dans leur salle de classe.

La décentralisation du financement de l'éducation n'a fait qu'accroître les disparités régionales : trois quarts des illettrés sont dans des régions rurales et $72,7 \%$ d'entre eux sont des femmes, selon le recensement de 2000. Si l'on examine non plus le taux d'admission en primaire, mais la proportion d'élèves achevant en six ans sa formation primaire sans redoubler, les résultats du Tibet (41,24\%), du Ningxia $(49,83 \%)$, du Qinghai (53,94\%), du Hainan (54,76\%), du Gansu (55,38\%), du Guizhou (56,13\%) et du Sichuan $(57,68 \%)$ sont très en dessous de la moyenne nationale $(76,6 \%)^{27}$ : l'abandon des études est un phénomène -d'ampleur dans les régions défavorisées et les filles sont les premières à quitter l'école. En 2002, le taux de scolarisation des filles en primaire était beaucoup plus faible dans les provinces du Tibet (87,26\%), du Qinghai (93,73\%), du Chongqing (94,89\%), du Yunnan (95,75\%) et du Ningxia (96,51\%) qu'au niveau national où l'écart entre les sexes est minime (98,53\% pour les filles contre $98,62 \%$ pour les garçons) ${ }^{28}$. Les autorités estiment qu'en 2004 ce sont près de 2,3 millions d'enfants qui ont quitté l'école, parmi lesquels $70 \%$ de filles. Le taux d'abandon dans les zones rurales est de $2,45 \%$ pour le primaire et de $3,91 \%$ pour le collège ${ }^{29}$. Plusieurs facteurs expliquent ce phénomène ${ }^{30}$ : la pauvreté des familles, la pression des examens et la faible probabilité d'être admis au lycée voire à l'université, et l'impossibilité de financer ces études. S'ajoutent à cela un encadrement et un management éducatifs insuffisants ainsi qu'un manque d'intérêt pour des formations dont le contenu est souvent médiocre. Les enseignants des zones rurales sont moins bien formés et moins payés que ceux des régions urbaines ${ }^{31}$ : en 2001, la proportion d'enseignants ayant suivi des études supérieures était en ville de $40,94 \%$ pour le primaire et de $23,51 \%$ pour le collège, alors qu'en zone rurale elle était de $20,25 \%$ et 9,35\%. La presse se fait chaque année l'écho de centaines d'enseignants qui, las de leurs piètres conditions de travail, migrent vers de plus grosses villes.

En février 2006, le ministre de l'Éducation, M. Zhou Ji, a reconnu que « les ressources éducatives ne sont pas réparties équitablement. La disparité entre les écoles et les régions, entre les villes et la campagne, s'accroît chaque jour ${ }^{32}$.» En 1998, le budget alloué par enfant à Shanghai était dix fois supérieur à celui donné par la province centrale du Henan; en 2005, le rapport est de cinquante par enfant entre une école de Shanghai et une école rurale du Henan ${ }^{33}$. Ce constat de l'inégalité croissante dans le droit d'accès à l'éducation a conduit à amender en février 2006 la loi de 1986. Depuis la rentrée 2006, les écoles rurales des provinces de l'Ouest peuvent être soutenues 
financièrement par les autorités centrales et provinciales afin d'assurer la gratuité effective des neuf ans d'études obligatoires. Les écoles et collèges ruraux ne peuvent percevoir de l'argent des familles que pour l'achat des livres et des cahiers d'exercices ainsi que pour la cantine et l'internat. Des allocations spécifiques devraient être versées aux familles les plus pauvres pour couvrir ces dépenses. Cette mesure devrait s'étendre en 2007 aux zones rurales des provinces centrales et de l'Est. Le soutien des autorités centrales et provinciales en faveur de l'éducation dans les campagnes (où vit $60 \%$ de la population chinoise) devrait être renforcé ${ }^{34}$. L'objectif est de descendre en 2010 en deçà de $2 \%$ d'illettrisme chez les $15-50$ ans, et de $5 \%$ sur l'ensemble de la population. ${ }^{35}$

On peut néanmoins se demander si ces mesures suffiront car face au coût élevé des études, beaucoup de familles rurales s'interrogent sur l'intérêt économique qu'il y a pour leurs enfants à étudier . Le fossé qui s'est creusé ces dernières années entre la ville et la campagne incite de plus en plus de ruraux à émigrer à la ville. Le marché du travail qualifié étant quasi inexistant à la campagne, la tentation est grande pour un jeune que l'âpreté de la vie agricole rebute de partir vers les centres urbains où l'on trouve facilement du travail non qualifié mieux rémunéré36.

\section{Développement de l'enseignement privé}

L'enseignement privé en Chine a une longue histoire et existait déjà à l'époque des Printemps et Automne (770-475 av. J.-C.) et de la dynastie Han (206 av. J.-C-220 apr. J.C.). Les écoles privées « modernes » furent ouvertes par les missionnaires à la suite du traité de Nankin signé en 1842 entre le gouvernement des Qing et les puissances occidentales à l'issue de la première guerre de l'Opium. Jusqu'au milieu du XX. siècle, l'enseignement privé s'est beaucoup développé : en 1950, 77 universités privées (sur un total de 227) accueillaient $40 \%$ des étudiants ${ }^{37}$. En 1951, sous l'influence du modèle soviétique et des radicaux du PCC, les établissements privés furent fermés ou transformés en établissements publics. De 1952 à 1978, il n'y eut en Chine que des établissements éducatifs "gérés par le peuple ", c'est-à-dire par une communauté de personnes ou une organisation collective.

Avec l'ouverture économique du début des années 1980, les autorités ont rouvert la porte à l'enseignement privé. L'article 19 de la Constitution de 1982 stipule que « l'État encourage les organisations de l'économie collective, les organisations des entreprises et des institutions de l'État, ainsi que d'autres forces de la société à créer [...] des œuvres d'éducation de tout genre ${ }^{38} »$. En 1992, après une tournée de Deng Xiaoping dans le Sud de la Chine, le PCC a adopté lors de son XIV.Congrès le principe de "l'économie socialiste de marché »: pour faire face aux enjeux de la modernisation, le développement de l'économie et de l'éducation devait s'appuyer sur d'autres forces que celles de l'État. L'article 25 de la loi sur l'éducation de 1995 précise que « l'État encourage les entreprises, les institutions, les organisations publiques et sociales ainsi que les citoyens à fonder et gérer des écoles ou d'autres institutions de formation ». La loi de 2002 sur l'enseignement non gouvernemental autorise le développement d'initiatives privées pour les cycles d'enseignement qui ne sont pas obligatoires afin de mieux répondre à des demandes sociales particulières. 

ont ouvert la porte à une composante privée : parallèlement aux quotas d'élèves ayant réussi les examens de sélection, étaient admis, hors contingent, des élèves ayant échoué à la sélection mais qui, contre le paiement d'un droit d'inscription élevé, pouvaient suivre la même formation ou une formation parallèle. Pour ceux qui avaient connu l'échec et avaient des moyens financiers, le privé constituait une seconde chance de poursuivre leurs études. C'est ainsi que les institutions privées se développèrent dans les grandes villes des provinces côtières. Au début des années 1990, l'émergence d'une catégorie sociale aux revenus élevés qui avait largement profité de la croissance économique ainsi que les effets de la politique de l'enfant unique élargirent la demande vis-à-vis d'établissements ayant de meilleures prestations que celles du public ${ }^{40}$. La transition de l'économie planifiée vers l'économie de marché induisit également une forte demande de formation professionnelle à laquelle le secteur privé sut rapidement répondre. Enfin pour des publics fragiles tels que les enfants de migrants qui ont rejoint leurs parents mais ne disposent pas d'un permis de résidence (hukou) autorisant la scolarité dans une école publique de la ville où ils vivent, ainsi que pour les enfants illégitimes qui n'ont pas de statut légal, les écoles privées restent la seule solution ${ }^{41}$.

\section{L'enseignement supérieur}

\section{Des origines à l'ouverture}

Les premières universités chinoises modernes furent créées sur le modèle japonais ou américain aux alentours de 1900. En 1952, les universités privées ou financées par les missions étrangères furent transformées en établissements publics ou fermées ${ }^{42}$. Dans un contexte d'économie centralisée planifiée, les autorités adoptèrent alors le modèle soviétique : la plupart des établissements supérieurs se spécialisèrent et leur première tâche fut de former, selon les objectifs du plan, des diplômés ensuite recrutés par les entreprises d'État - elles-mêmes sous l'autorité des ministères ou des gouvernements provinciaux. Dès lors, mis à part les universités les plus réputées qui conservèrent 
quelques laboratoires, les établissements ne se soucièrent plus de recherche, cette activité étant regroupée au sein de l'Académie des sciences de Chine. Parmi les 1032 établissements supérieurs que l'on décomptait dans les années 1950, 35 relevaient directement de la commission d'État de l'Éducation, et 311 de 61 ministères. Les 686 autres étaient sous l'autorité des gouvernements provinciaux par secteur d'activité (industrie légère ou lourde, pétrole, mines, agriculture, etc.). Cette segmentation à outrance eut trois conséquences majeures : a) les formations furent hyperspécialisées puisque leur contenu était fixé par des ministères ou des bureaux provinciaux couvrant eux-mêmes un spectre très étroit; b) la taille des établissements resta petite (les besoins humains pour des compétences aussi pointues étant limités) ; c) beaucoup de moyens matériels et humains furent gaspillés (équipements sous-utilisés, effectifs enseignants disproportionnés par rapport au petit nombre d'étudiants, absence de lien entre l'enseignement et la recherche).

Durant la Révolution culturelle (1966-1976), les luttes politiques prirent le pas sur l'éducation et il fallut attendre l'hiver 1977 pour la reprise du gaokao. Sur 5,7 millions de candidats, seuls $273000(4,8 \%)$ furent admis. Ces chanceux bénéficièrent d'études gratuites et d'une petite indemnité de frais de vie, ainsi que de l'assurance d'un emploi dans une entreprise d'État à la sortie. En revanche, pour les exclus du système, le gâchis fut énorme.

Le mouvement d'ouverture lancé en 1978 par Deng Xiaoping toucha d'abord les campagnes puis se propagea aux villes. L'essor du secteur privé dans le domaine des services et de l'industrie légère accrut le besoin en personnel efficace et productif. Du coup les universités se multiplièrent (1 075 en 1988 contre 633 en 1979) et la durée des formations passa de deux à trois ans. Mais l'efficacité de ces établissements dont l'effectif ne dépassait que rarement 2000 étudiants restait médiocre: dans les années 1980, le ratio étudiants/ enseignant était proche de 4 pour 1 (contre une moyenne internationale de 10 à 12 pour 1), la charge horaire d'enseignement hebdomadaire était de 4,8 heures pour les enseignants (contre une fourchette de 9 à 12 heures dans les autres pays de l'aire Asie-Pacifique). En 1986, on dénombrait 15804 spécialités pour 1,8 million d'étudiants (soit un recrutement moyen de 119 étudiants par spécialité). L'émergence de l'économie socialiste de marché conduisit, au milieu des années 1990, à un bouleversement du paysage universitaire ${ }^{43}$.

\section{La massification de l'enseignement supérieur}

30 Pour mettre fin à l'hyperspécialisation et aux innombrables doublons locaux hérités de l'économie planifiée, le ministère de l'éducation lança entre 1995 et 2002, en concertation avec les autorités locales, une grande vague de regroupements d'établissements. Il s'agissait de réaliser des économies d'échelle, de faire émerger au niveau de la Chine une carte des formations et des pôles d'excellence, et de mettre en place une politique d'investissements indispensable pour accroître les capacités d'accueil. En 2000, près de 612 établissements supérieurs avaient fusionné pour donner naissance à 250 établissements de plus grande taille ${ }^{44}$. Les universités jouent désormais un rôle moteur dans le développement régional ${ }^{45}$. C'est ainsi qu'en périphérie de grandes métropoles ont émergé ces dernières années des méga-campus accueillant 50000 à 100000 étudiants. 
31 Les établissements sont désormais placés sous l'autorité du ministère de l'Éducation pour ce qui relève des missions et des principes éducatifs, et sous celle d'une autorité centrale ou locale pour leur financement. Les établissements supérieurs dénombrés en 2004 (hors universités à distance) se répartissent ainsi ${ }^{46}$ :

- - universités d'excellence qui dépendent du ministère de l'Éducation (73) ou d'un autre ministère (38) ;

- - universités relevant d'un gouvernement provincial ou municipal (1394) ;

- - universités publiques qui ne relèvent ni du gouvernement central ni d'un gouvernement local (226);

- - universités privées qui sont supervisées au niveau provincial ou municipal mais ne bénéficient d'aucun financement public (1 187).

Pour accompagner le développement des établissements, les droits d'inscription ont été généralisés à partir de 1996. Le montant de ces droits, qui couvrent de 20 à $25 \%$ du budget des établissements, est fixé par le ministère de l'Éducation d'après le coût théorique de chaque formation. Il atteint couramment 6000 à 7000 yuans annuels (600 à 700 euros) pour des formations générales, c'est-à-dire plus de six mois du revenu moyen d'un résident urbain et deux ans et demi de celui d'un paysan. Les formations scientifiques ou informatiques peuvent coûter jusqu'à 10000 yuans (1 000 euros).

Grâce à une politique volontariste, les effectifs étudiants ont connu un quasi-quadruplement entre 1998 et 2004. Du fait de la pyramide des âges, la cohorte la plus nombreuse est attendue à l'université en 2008 ; durant les années suivantes la pression démographique diminuera. Avec un taux d'accès aux études supérieures de $19 \%$ la Chine a comblé une partie de son retard, mais reste loin derrière les pays développés où 50 à $70 \%$ des 18-22 ans suivent des études supérieures.

\section{Réformer le management en vue de l'excellence}

Les activités de services destinées aux étudiants des campus (magasins, banques, alimentations, hôpitaux, etc.) ont été supprimées ou externalisées. La réforme donne une plus grande autonomie aux universités, notamment pour le recrutement des étudiants, l'organisation des facultés et départements, le contenu précis des formations, le développement de la recherche, la coopération internationale, la gestion du personnel, l'affectation des ressources financières. En contrepartie de la justification a posteriori de leurs dépenses, elles disposent d'une plus grande liberté de décision quant à l'affectation de leurs fonds. Cette approche responsabilise les établissements et favorise les initiatives locales même si c'est toujours le ministère de l'Éducation qui nomme le président d'université, fixe le montant des frais d'inscription et arrête la liste des spécialités donnant droit à des diplômes. La réduction du personnel administratif et de service a permis de revaloriser le salaire des enseignants. Le recrutement et la rémunération de ces derniers dépendent à présent de la politique de l'établissement et des responsabilités qu'ils assument (encadrement doctoral, direction d'équipe, publications internationales, etc.). L'évaluation des enseignements par les collègues et par les étudiants devient de plus en plus courante. La publication dans la presse chinoise et anglophone d'annonces de vacances de postes afin d'attirer des universitaires d'origine chinoise exerçant à l'étranger ou dans d'autres universités chinoises atteste de l'émergence d'un marché de la mobilité au sein du corps enseignant. 

autour de thèmes prioritaires de formation et de recherche, afin de doter le pays des compétences scientifiques et technologiques requises pour poursuivre son développement dans un contexte concurrentiel. Pour obtenir et conserver ce label, les universités sont évaluées et négocient avec leur autorité de tutelle les objectifs à atteindre. Ce projet, qui regroupe 94 établissements supérieurs (c'est-à-dire moins de $6 \%$ des établissements publics) inégalement répartis sur le territoire chinois, a abouti à la mise en place nationale d'un réseau informatique à haut débit dédié à l'enseignement et à la recherche. Les universités labellisées « 211 » regroupent $96 \%$ des laboratoires clés d'état, $85 \%$ des disciplines clés, $87 \%$ des enseignants qui interviennent au niveau doctoral, $32 \%$ des étudiants en benke (bac +4$), 69 \%$ des étudiants en master, $84 \%$ des étudiants en doctorat. Elles disposent de $54 \%$ des équipements de recherche et drainent $72 \%$ des fonds de la recherche scientifique chinoise. Le "projet 985 ", regroupe, depuis 1998, les 9 meilleures « universités 211 » avec l'idée de les hisser dans le palmarès mondial d'ici vingt à trente ans.

De plus en plus d'universités chinoises s'associent avec des universités étrangères pour co-délivrer sur le sol chinois des diplômes reconnus à l'étranger. Depuis 1999, le nombre d'étudiants poursuivant à leurs frais des études à l'étranger a fortement augmenté. Près de 400000 Chinois étudient à l'étranger, ce qui fait de la Chine la première source d'étudiants étrangers. Les destinations préférées sont les États-Unis, le Royaume-Uni, le Japon, l'Australie, la Nouvelle-Zélande, l'Allemagne, le Canada, la Corée et la France. En 2004, 114682 Chinois sont partis étudier à l'étranger, mais en dépit de mesures incitatives seuls 24726 étudiants sont revenus au pays, c'est-à-dire un peu plus du cinquième de ceux qui sont partis la même année.

Entre 2002 et 2004, plusieurs des universités réputées ont fait une réforme de la gestion du personnel enseignant (dont l'université de Pékin, de Tsinghua, de Nankin, de Fudan et Sun Yat-sen). L'objectif était de mettre en place des procédures de recrutement et de promotion du personnel enseignant qui améliorent la compétitivité face aux meilleures universités mondiales. L'écho médiatique soulevé par ces réformes a permis de constater que s'il y a un consensus en Chine sur la légitimité de cet objectif, les vues sur les façons d'y parvenir divergent notablement. Les grandes lignes des mesures adoptées portent sur l'introduction de la concurrence et de la mobilité dans la gestion des carrières des enseignants, et s'inspirent $d u$ fonctionnement des universités américaines. Les postes de professeurs titulaires sont désormais réservés à ceux qui feront la preuve de leurs compétences. Les maîtres de conférences et les assistants nouvellement recrutés le seront sur des contrats à durée déterminée, renouvelables un nombre limité de fois. Les recrutements sont largement ouverts sur l'extérieur et les profils de poste sont diffusés à l'échelle nationale et internationale. L'évaluation des activités d'enseignement et de recherche par une commission scientifique de l'établissement devient systématique. Les détracteurs de ces changements ont affirmé que les universités chinoises souffrent d'abord d'une hypertrophie de l'administration (de fait, la proportion des enseignants à temps plein sur l'ensemble du personnel universitaire est passée de $39 \%$ en 1999 à $53 \%$ en 2004). Des voix se sont élevées pour dire que si l'université est encore l'un des rares secteurs de la société chinoise à échapper à la concurrence du marché, c'est justement pour sauvegarder la liberté académique des enseignants, indispensable au développement de la connaissance et de la recherche. D'autres ont souligné que le placage de modalités empruntées aux 
universités américaines sur des universités chinoises, construites sur d'autres bases historiques et culturelles, risque au contraire d'affaiblir ces dernières. Dans la réalité, la question sous-jacente porte sur la prééminence accordée, au sein des établissements, aux structures administratives et politiques. Le pouvoir dont jouit aujourd'hui l'administration est en contradiction avec la mise en place de critères transparents d'évaluation académique et scientifique. Un président d'université a-t-il l'autonomie et l'autorité suffisantes pour piloter l'enseignement et la recherche au sein de son établissement alors qu'il est sous l'autorité du secrétaire général du Parti communiste et que le PCC doublonne l'organigramme de l'université à chaque niveau ? L'innovation et la créativité scientifique sont-elles compatibles avec le contrôle politique étroit que les organes du Parti exercent sur tous les aspects de la vie universitaire?

En privilégiant le quantitatif sur le qualitatif, c'est-à-dire en accroissant brutalement les effectifs étudiants sans s'être attelé au préalable à la réforme du système d'évaluation, à la lutte contre la fraude et le plagiat qui sont endémiques, à la formation d'enseignants capables non seulement de développer des enseignements centrés sur les apprenants mais également de mener des recherches dans des laboratoires où régnerait la liberté de penser, les autorités ont pris le risque de ne proposer que des demi-réformes qui ne suffiront peut-être pas à endiguer la fuite des cerveaux à l'étranger et à hisser les universités chinoises dans le palmarès mondial.

\section{Les défis des prochaines années}

La politique éducative défendue par Pékin ces dernières années a privilégié les investissements matériels au détriment du capital humain. Une comparaison avec l'Inde montre que la Chine n'investit pas assez dans l'éducation de base, notamment dans les zones rurales ${ }^{47}$. Pour les familles aux revenus modestes, les coûts de scolarisation sont très lourds. Les autorités auront à prendre des mesures réduisant les disparités régionales et garantissant un vrai droit à l'éducation pour éviter que le lieu de naissance ou de résidence ne devienne un frein à la scolarisation. À défaut, les enfants qui vivent dans les zones rurales laissées pour compte de la croissance ou les enfants de migrants sont socialement condamnés à devenir des citoyens de seconde classe.

Beaucoup de familles se sont endettées pour financer les études secondaires ou universitaires de leur progéniture. Avec la massification des flux, l'obtention d'un premier emploi passe souvent par l'acceptation d'une situation sous-payée pour laquelle le jeune est surdiplômé. Face à la dépréciation de leurs diplômes sur le marché du travail et à la concurrence avec les migrants, beaucoup de jeunes ont un sentiment de frustration. Ils sont également déçus de constater que les relations personnelles et l'origine sociale restent déterminantes pour trouver une place. Si cette situation devait perdurer, elle n'encouragerait pas à investir sur le capital humain ${ }^{48}$. En 2006, le marché de l'emploi s'est tendu: en mai, un rapport de la Commission nationale du développement et des réformes annonçait que près de $60 \%$ des diplômés de l'année ne trouveraient pas de poste à la sortie de l'universitét ${ }^{4}$. Près de 4,13 millions de diplômés ont rejoint le marché du travail (22\% de plus qu'en 2005) alors que ce dernier ne peut en absorber que 1,66 million. Ces nouveaux venus s'ajoutent aux $28 \%$ des diplômés de l'année précédente qui sont toujours en recherche d'emploi. En 2005, le salaire mensuel moyen d'embauche d'un diplômé était de 1588 yuans (158 euros). Le salaire mensuel 
moyen en 2006 de ceux qui travaillent et sont titulaires d'une licence (bac+4) délivrée en 2005 est de 1132 yuans $^{50}$ (113 euros). En 2006, 47,1\% des diplômés ayant trouvé du travail ont un salaire mensuel inférieur à 1500 yuans (150 euros) $)^{51}$. Si l'on considère l'élévation du coût de la vie et, en particulier celui du logement dans les grandes villes, le pouvoir d'achat des salariés a baissé. Ni la pause décidée par les autorités pour 2006-2007 dans l'accroissement du recrutement des étudiants, ni les mesures d'incitation à travailler à l'Ouest ou dans les villes de taille moyennes ou petites ne suffiront à réduire cette pression car ces difficultés sont structurelles : la marchandisation et la massification de l'éducation se sont faites en grande partie au détriment de la qualitéét. Les formations dispensées ne sont pas assez appliquées et sont inadaptées aux demandes des entreprises - notamment étrangères - du secteur des services ou des technologies à forte valeur ajoutée, qui demain assureront une large part du développement chinois ${ }^{53}$. Il importe de développer l'enseignement professionnel secondaire et supérieur, et de veiller à une meilleure utilisation des ressources humaines en soutenant l'implantation d'emplois qualifiés dans les zones rurales et dans les provinces de l'Ouest ainsi qu'en libéralisant le marché du travail ${ }^{54}$.

41 L'amélioration de la pédagogie et des modalités d'étude est indispensable pour mieux répondre aux besoins des entreprises. Des cours sans interaction, limités à la lecture à haute voix d'un manuel, pour lesquels la production écrite repose surtout sur la compilation et le plagiat, où la préparation des évaluations est fondée sur des apprentissages par cœur ne sont pas la meilleure voie pour développer la créativité, l'esprit critique, l'adaptabilité et les capacités de communication. Le système actuel de sélection fait subir aux élèves et aux établissements une très forte pression et pousse à un bachotage qui étouffe les talents novateurs ${ }^{55}$ : la vie de millions de lycéens se joue sur un coup de dés (les deux jours d'épreuves du concours national d'entrée à l'université). Pour ceux qui échouent, il n'y a pratiquement pas de seconde chance. Pour ceux qui réussissent, le choix de la formation se fait sans une véritable orientation préalable et sans vérifier l'adéquation du parcours de formation aux capacités ou à la curiosité des intéressés. Ici ou là, il y a bien eu quelques expériences pilotes de recrutement à base d'entretiens, mais cela concerne quelques centaines d'étudiants au plus ${ }^{56}$.

Les questions d'éducation occupent de plus en plus de place au sein de la société chinoise : toutes les familles s'intéressent au devenir de leur enfant et ont le souci légitime d'un accès équitable à tous les niveaux d'éducation. Domaine éminemment social, l'éducation est un révélateur des contradictions de ce que les autorités continuent d'appeler le "socialisme à la chinoise ». Les manifestations étudiantes de l'année 2005-2006 témoignent que les autorités auront à prendre toute la mesure des fortes attentes de la population concernant l'éducation si elles veulent se prémunir de ce risque d'instabilité sociale ${ }^{57}$. 


\section{BIBLIOGRAPHIE}

- BILleter J.-F., Contre François Jullien, Paris, Éditions Allia, 2006.

- CHEN D. Y., « China Mass Higher Edu-cation : Problem, Analysis, and Solu-tions », Asia Pacific Education Review, vol. 5, n 1, 2004, p. 23-33.

- CHEN D. Y., « China's Large Scale University Mer-gers in Retrospect », Chinese Cross Currents, vol. 2, n 3, juillet-septembre 2005, p. 64-75.

- CHEN Y., «The Reforms of Secondary Edu-ca-tion in China ", Chinese Cross Currents, vol. 2, $\mathrm{n}^{\circ}$ 3, juillet-septembre 2005, p. 42-63.

- CHEN Xin, « Social Changes and the Revival of Liberal Education in China since the 1990s », Asia Pacific Education Review, vol. 5, n 1, 2004, p. 1-13.

- CHENG H. et B. DeLany, «Quality Edu-cation and Social Stratification : The Paradox of Private Schooling in China », Current Issues in Comparative Education, Teachers College, Columbia University, 2002, p. 48-56.

- FROISSART C., « Les aléas du droit à l'éducation en Chine ", Perspectives chinoises, $\mathrm{n}^{\circ}$ 77, mai-juin 2003, p. 10.

- GERNET J., L'intelligence de la Chine, le social et le mental, Paris, Gallimard, 1994.

- GRENIÉ M. et A. BELOTEL-GRENIÉ, « Edu-cational Reforms in China », Chinese Cross Currents, vol. 2, $n^{\circ} 3$, juillet-septembre 2005, p. 8-31.

- HECKMAN J. J., « China's Investment in Human Capital », Economic Development and Cultural Change, vol. 51, 2003, p. 795-804.

- HECKMAN J. J, « China's Human Capital Investment », China Economic Review, 16:1, 2005, p. 50-70.

- HUI L., « Chinese cultural schema of Edu-cation : Implications for communication between Chinese students and Australian educators ", Issues in Educational Research, 15 (1), 2005, p. 17-36.

- KANG O., "Higher Education Reform in China Today », Policy Futures in Education, vol. 2, n 1, 2004, p. 141-149.

- MERLE A. et M. SZTANKE, Étudiants chinois : qui sont les élites de demain ? Paris, Autrement, 2006.

- LIU F., « Basic Education in China's Rural Areas : a Legal Obligation or an Individual Choice?» International Journal of Educational Development, 24, 2004, p. 5-21.

- ROCCA J.-L., La condition chinoise : capitalisme, mise au travail et resistances dans la Chine des réformes, Paris, Karthala, 2006.

- stockman N., Understanding Chinese society, Malden, Polity Press, 2000.

- TSANG MUN C., « School Choice in People Republic of China », Occasional Paper No. 9, Teachers College, Columbia University, novembre 2000.

- WANG Y. et KE X. « A Research Note on China's higher Education », Journal of Contem-porary China, 12 (36), août 2003, p. 587-596.

- XIANG X., « Academic Management and Administration System Reform in Higher Education Institutions », Frontiers of Edu-ca-tion in China, vol. 1, n 1, janvier 2006, p. 70-78. 
- XU Z., « An Overview of Private Education Development in Modern China », Education Policy Analysis Archives, vol. 10, n 47, 2002. Accessible en ligne sur http://epaa.asu. edu/epaa/v10n47/.

- YANG D., China's Education Blue Book (2003), Beijing, Higher Education Press, 2004.

«OECD Review of Financing and Quality Assurance Reforms in Higher Education in The People's Republic of China », OECD EDU/EC/NME, (2004)1, 25 octobre 2004.

\section{NOTES}

1. Le Monde, 20 juin 2006 et The Economist, 10 août 2006.

2. Voir à ce propos le passionnant essai de Jean-Louis Rocca, La condition chinoise, Paris, Karthala, 2006.

3. James J. Heckman " China's investment in human capital », Economic Development and Cultural Change, vol. 51, 2003, p. 795-804 ; James J. Heckman, « China's Human Capital Investment », China Economic Review, 16:1, 2005, p. 50-70.

4. Jacques Gernet, L'intelligence de la Chine, le social et le mental, Paris, Gallimard, 1994, p. 103.

5. Jacques Gernet, op. cit., p. 103.

6. Leng Hui, «Chinese cultural schema of Education : Implications for Communication between Chinese Students and Australian Educators ", Issues in Educational Research, 15 (1), 2005, p. 17-36 ; Jacques Gernet, op. cit., p. 115.

7. Jacques Gernet, op. cit., p. 125.

8. Norman Stockman, Understanding Chinese Society, Malden, Polity Press, 2000, p. 150-151.

9. Jean-François Billeter, Contre François Jullien, Paris, Éditions Allia, 2006, p. 18.

10. Norman Stockman, op. cit., p. 159.

11. Qingjun Zhuo, "Mao Zedong», Perspectives Revue trimestrielle d'éducation comparée, Paris, Unesco, vol. XXIV, n 1-2, 1994, p. 97-110.

12. Jacques Gernet, op. cit., p. 114.

13. Ce concept a été élevé au rang de pensée officielle par le secrétaire général du PCC, M. Hu Jintao, à la suite de la sixième session plénière du $\mathrm{XVI}^{\mathrm{e}}$ Comité central qui s'est achevé le 11 octobre 2006. Voir l'article de Bruno Philip, «Hu Jintao promet l'“harmonie sociale” contre les inégalités et la corruption en Chine », Le Monde, 13 octobre 2006.

14. China Statistical Yearbook 2004, Beijing, China Statistics Press, 2005 et Educational statistics yearbook of China 2004, Beijing, People's Education Press, 2005.

15. A Brief Account of Education in People's Republic of China, Ministry of Education, The People's Republic of China, 2002 et Education in China, Ministry of Education, The People's Republic of China, 2002.

16. Ce concours est organisé les 7 et 8 juin chaque année dans tout le pays. Les élèves de dernière année de lycée sont évalués sur le chinois, l'anglais et les mathématiques, et doivent en plus choisir une épreuve de synthèse, littéraire (histoire, géographie ou politique) ou scientifique (chimie, physique, biologie) selon le parcours suivi au lycée. Le contenu de cette dernière épreuve varie selon les provinces. Certaines provinces ont fait le choix d'autoriser deux épreuves de synthèse, ce qui explique que la note maximale puisse varier. Des points supplémentaires peuvent être obtenus pour ceux qui présentent des épreuves spéciales (sport, musique...), pour ceux qui ont témoigné durant leur scolarité de qualités morales ou intellectuelles remarquables ou pour des élèves issus de minorités nationales. Chaque université applique ensuite son plan de recrutement selon l'origine des candidats: elle privilégie les candidats locaux, mais admet également un certain nombre de candidats issus d'autres provinces à condition qu'ils aient obtenus une note supérieure à la note exigée localement. Ce principe accentue fortement les inégalités entre les provinces côtières et les régions de l'Ouest : à niveau égal, un lycéen de Pékin 
ou de Shanghai, où les universités sont nombreuses, a beaucoup plus de chances d'être admis à l'université que quelqu'un du Xinjiang.

17. Sauf mention contraire, les statistiques citées dans cet article sont tirées de China Statistical Yearbook 2004, op. cit.

18. Ce total n'intègre pas les effectifs de l'enseignement supérieur pour adultes (4,19 millions d'étudiants) ni les formations supérieures assurées à distance via Internet (2,36 millions).

19. Constitution de la République populaire de Chine, Pékin, Éditions en langues étrangères, 1983.

20. La « loi sur l'enseignement obligatoire" du 12 avril 1986 (Compulsory Education Law of the People's Republic of China, Beijing, Law Press China, 2004), la « loi sur l'éducation » du 18 mars 1995 (Education Law of the People's Republic of China, Beijing, Law Press China, 2004), la «loi sur l'enseignement professionnel » du 15 mai 1996 (Vocational Education Law of the People's Republic of China, Beijing, Law Press China, 2002), la " loi sur l'enseignement supérieur » du 29 août 1998 (Higher Education Law of the People's Republic of China, Beijing, Law Press China, 2002), la « loi en faveur de l'enseignement privé " du 28 décembre 2002 (Law of the People's Republic of China on Promotion of Privately-run Schools, Beijing, Law Press China, 2002) et la " loi sur les écoles sinoétrangères de coopération" du 19 février 2003 (Regulation of the People's Republic of China on Chinese-Foreign Cooperation in Running Schools, Beijing, China Legal Publishing House, 2003).

21. C'est l'appellation du gouvernement chinois.

22. Yangbin Chen, "The Reforms of Secondary Education in China ", Chinese Cross Curent, juilletseptembre 2005, p. 42-63.

23. Zhao Huanxin, « China to Boost Spending on Education », China Daily, $1^{\mathrm{er}}$ mars 2006.

24. Ce chiffre est d'autant plus élevé qu'en théorie les neuf années d'éducation obligatoire sont gratuites.

25. "Chinese Students Face too much Educational Expenses », Xinhua (agence Chine nouvelle), 8 mars 2006.

26. Selon le ministère de l'Éducation, 93 \% des établissements primaires et secondaires disposent de telles entreprises.

27. Chiffres de 2002. Zhou Yixian et Zhang Yanshu, « Rural Education : Progress and Problems », China's Education Blue Book (2003), Beijing, Higher Education Press, 2004, p. 150-175.

28. Zhou Yixian et Zhang Yanshu, op. cit., p. 158.

29. Sun Shangwu, "Legislature Reviews Education Disparity », China Daily, 27 février 2006.

30. Fengshi Liu, «Basic Education in China's Rural Areas: a Legal Obligation or an Individual Choice? » International Journal of Educational Development, 24, 2004, p. 5-21.

31. Zhou Yixian et Zhang Yanshu, op. cit.

32. «Compulsory Education Law under Amendment », Xinhua, 26 février 2006.

33. Ibid.

34. Wen Jiabao, «Rapport d'activité du gouvernement », présenté le 5 mars 2006 à la quatrième session de la $\mathrm{X}^{\mathrm{e}}$ Assemblée populaire nationale.

35. Fengshi Liu, «Basic Education in China's Rural Areas : a Legal Obligation or an Individual Choice ?» International Journal of Educational Development, 24, 2004, p. 5-21.

36. Un migrant, manœuvre sur un chantier à Pékin ou Shenzhen, gagne entre 800 et 1200 yuans (80 à 120 euros) par mois. China Daily, 7 juin 2006.

37. Zeyu Xu, "An Overview of Private Education Development in Modern China », Education Policy Analysis Archives, vol. 10, $\mathrm{n}^{\circ}$ 47, 2002.

38. Constitution de la République populaire de Chine, op. cit.

39. China Statistical Yearbook 2004, op. cit.

40. Haojing Cheng et Brian DeLany, «Quality Education and Social Stratification : the Paradox of Private Schooling in China », Current Issues in Comparative Education, Teachers College, Columbia University, 2002, p. 48-56. 
41. Chloé Froissart, «Les aléas du droit à l'éducation en Chine », Perspectives chinoises, $\mathrm{n}^{\circ}$ 77, maijuin 2003, p. 10.

42. Ma Wanhua, « Economic Reform and Higher Education in China », CIDE Ocassional Papers Series : Higher Education, CIDE Contributions No. 2, juillet 2003, 24 p. ; Ouyang Kang, « Higher Education Reform in China Today », Policy Futures in Education, vol. 2, n 1, 2004, p. 141-149.

43. "OECD Review of Financing and Quality Assurance Reforms in Higher Education in The People's Republic of China », OECD EDU/EC/NME, (2004)1, 25 octobre 2004, 66 p.

44. David Chen Yunchao, "China's Large Scale University Mergers in Retrospect ", Chinese Cross Curent, juillet-septembre 2005, p. 64-75.

45. K. Mohrman, «Higher Education Reform in Mainland Chinese Universities : An American's Perspective ", Hong Kong American Centre, Chinese University of Hong Kong, juillet 2003, 98 p.

46. China Statistical Yearbook 2004, op. cit.

47. Yasheng Huang, "China Could Learn from India's Slow and Quiet Rise », The Financial Times, 27 janvier 2006.

48. James J. Heckman, art. cit.

49. "Millions of Graduates Facing Unemployment », Xinhua, 7 mai 2006.

50. Valeur à comparer aux 800 à 1200 yuans d'un migrant travaillant sur un chantier à Pékin ou Shenzhen.

51. Liu Shinan, « Improved Education Standards Required », China Daily, 7 juin 2006.

52. Emily Patterson, «Drowning in the Talent Pool», China Daily, 12 juin 2006 ; Liu Jie, « What Graduates Want », China Daily, 31 juillet 2006.

53. "Reform Curriculum to Help Graduates Find Jobs ", China Daily, 3 juin 2005 ; Diana Farrell et Andrew J. Grant, « China's Looming Talent Shortage », The McKinsey Quarterly, n 4, 2005, p. 71-79; Lindsay Beck, « Despite Boom, China Graduates Face Job Crunch », Reuters, 4 juillet 2006.

54. "China Suffers a Big Loss in Human Resource Utilization », China Daily, 26 juillet 2006.

55. Raymond Zhou, « Don't Let Gaokao Seal your Fate », China Daily, 10 juin 2006.

56. «Interview, New Trial for Universities' Enrollment », Xinhua, 5 avril 2006.

57. Des manifestations étudiantes violentes ont éclaté en décembre 2005 à l'Institut de l'information de l'Est à Dalian, en juin 2006 à l'université de Zhengzhou, à l'université du Sichuan ainsi qu'à l'Institut de Jiujiang dans le Jiangsu, et enfin le 25 octobre dernier dans le Jiangxi.

INDEX

Index géographique : Chine

Keywords : China, education system, elementary education, universities

Mots-clés : système éducatif, enseignement primaire, université

\section{AUTEURS}

\section{MICHEL GRENIÉ}

Michel Grenié est maître de conférences de linguistique à l'université de La Rochelle. De 1999 à 2003, il a été chargé de la coopération linguistique et éducative au service de coopération et d'action culturelle auprès de l'Ambassade de France à Pékin. À ce titre, il 
a visité et mis en place des coopérations avec de nombreux établissements secondaires et supérieurs chinois dans un très grand nombre de provinces. Il poursuit désormais des recherches sur l'éducation en Chine.

mgrenie@univ-lr.fr

\section{AGNÈS BELOTEL-GRENIÉ}

Agnès Belotel-Grenié est linguiste et enseignante de chinois. Elle a effectué pendant plusieurs années des recherches au sein du laboratoire de linguistique de l'Académie des sciences sociales de Chine à Pékin.

abelotel_grenie@hotmail.com 